\title{
A novel prophylactic Chinese parachute ankle brace
}

\author{
Xi Zhou ${ }^{1 \#}$, Di Wu ${ }^{1 \#}$, Xiangdong Wu ${ }^{1 \#}$, Zhengyao Li $^{2 \#}$, Bin Yan $^{3}$, Leilei Liang ${ }^{4}, \mathrm{Yu} \mathrm{He}^{2}$, Yong Liu ${ }^{1}$ \\ ${ }^{1}$ Department of Orthopedic Surgery, Peking Union Medical College Hospital, Peking Union Medical College and Chinese Academy of Medical \\ Sciences, Beijing, China; ${ }^{2}$ Department of Plastic Surgery, Plastic Surgery Hospital, Peking Union Medical College and Chinese Academy of Medical \\ Sciences, Beijing, China; ${ }^{3}$ Department of traditional Chinese medicine, Peking Union Medical College Hospital, Peking Union Medical College and \\ Chinese Academy of Medical Sciences, Beijing, China; ${ }^{4}$ Department of General Surgery, Tongren Hospital, Shanghai Jiao Tong University School of \\ Medicine, Shanghai, China \\ Contributions: (I) Conception and design: Y Liu, Y He; (II) Administrative support: Y Liu; (III) Provision of study materials or patients: X Zhou, D \\ Wu; (IV) Collection and assembly of data: D Wu, X Wu, Z Li; (V) Data analysis and interpretation: B Yan, L Liang; (VI) Manuscript writing: All \\ authors; (VII) Final approval of manuscript: All authors. \\ "These authors contributed equally to this work. \\ Correspondence to: Yong Liu. Department of Orthopedic Surgery, Peking Union Medical College Hospital, Peking Union Medical College and \\ Chinese Academy of Medical Sciences, No. 1 Shuaifuyuan Rd., Beijing 100730, China. Email: liuyongpumc@163.com; Yu He. Department of \\ Plastic Surgery, Plastic Surgery Hospital, Peking Union Medical College and Chinese Academy of Medical Sciences, No. 1 Shuaifuyuan Rd., Beijing \\ 100730, China. Email: heyuworkmail@sina.com.
}

Background: The objective is to compare the effects of a self-designed and self-manufactured novel prophylactic ankle brace [Chinese parachute ankle brace (CPAB)] and two ordinary ankle braces on the ankle joint during a half-squat parachute landing (HSPL) via biomechanical assessment.

Methods: Twenty elite paratroopers were in four different conditions: no brace, elastic brace, semi-rigid brace, and CPAB. Each participant was instructed to jump off a platform with three different heights, 40, 80, and $120 \mathrm{~cm}$, and land on the force plate in a half-squat posture. The vertical ground reaction forces (vGRFs), joint angles, moments, powers, and works were calculated. After the experiment, every participant completed the questionnaires designed for this study.

Results: Increasing the dropping height increased all of the parameters significantly $(\mathrm{P}<0.01)$, except for time to peak vGRF (T-PvGRF). Applying three braces can all slightly increase vGRF $(\mathrm{P}=0.237)$ and reduce T-PvGRF by $6-10 \mathrm{~ms}$, as well as decrease the joint angles, velocities, and moments on the sagittal and coronal planes. Wearing $\mathrm{CPAB}$ and a semi-rigid brace more efficiently restricted dorsiflexion and inversion $(\mathrm{P}<0.05)$, and they both significantly reduced ankle work $(\mathrm{t}=5.107, \mathrm{P}<0.01 ; \mathrm{t}=3.331, \mathrm{P}<0.01)$ and peak power $(\mathrm{t}=7.237, \mathrm{P}<0.01 ; \mathrm{t}=6.711, \mathrm{P}<0.01)$ at $120 \mathrm{~cm}$. The total scores from low-to-high were semi-rigid brace (19.20 \pm 2.99$)$, elastic brace $(21.91 \pm 3.25)$, and CPAB $(23.37 \pm 3.08)$.

Conclusions: The $\mathrm{CPAB}$ was more effective at restricting ankle joint motion on the coronal and sagittal planes than the other two prophylactic ankle braces. Therefore, the CPAB had the advantages of a novel appearance, high efficiency, and superior comfort, providing a reliable choice for parachute jumping and training in China.

Keywords: Ankle joint; prophylactic braces; half-squat parachute landing (HSPL); biomechanics; subjective scoring

Submitted Jun 25, 2020. Accepted for publication Nov 08, 2020.

doi: 10.21037/atm-20-4937

View this article at: http://dx.doi.org/10.21037/atm-20-4937 


\section{Introduction}

Landing injuries are the most frequent of all parachuting injuries and ankle joint injuries are the most common landing injury (1). The causes of ankle injury during parachute landing are complex. Ekeland conducted a retrospective analysis of 4,499 parachuting landing injuries and found that about $71 \%$ of injuries were caused by an incorrect landing posture (2). According to the investigation conducted by Dhar on 150 hospitalized patients with parachuting injuries, $53 \%$ of the parachuting injuries occurred during the landing stage, which were caused by inappropriate landing methods, and other influencing factors included improper cabin exit, uneven ground, windy weather, and inappropriate parachuting operation (3). The half-squat parachute landing (HSPL) posture has been adopted by Chinese paratroops $(4,5)$. When compared with the sideways roll parachute landing fall, the HSPL method may reduce the probability of asynchronous landings on either the left or right foot (6). In this maneuver, the upper body is in a neutral stance, and the lower extremities are in a half-squat position, with the legs slightly bent and extended forward. Bilateral knees, medial malleoli, and feet are kept tight, and the feet should be parallel with the ground (7). HSPL is characterized by actively and deeply flexing the joints of the lower extremities after initial contact, thereby prolonging impact absorption by the body segments and preventing potential injury $(4,5,7)$.

Ankle braces can reduce ankle injuries by $61 \%$, while this figure increases to $79 \%$ for people with old wounds (8). According to the application method and appearance design, available ankle braces are mainly divided into three categories: fixed slip-on, bandage winding, and bandage slip-on. Moreover, they can also be divided into the external types, internal type, and internal and external dual-use type (9). The inconvenient external ankle brace is generally made of rigid polyethylene material, which is bulky and uncomfortable. The internal ankle brace is usually a sports product with good elasticity, but it cannot prevent excessive eversion and inversion of the ankle joint. The dual-use ankle brace is rare currently (10).

The protective performance of the ankle brace depends on not only its material, structure, and application method, but also its protective effect on the biomechanical mechanism of the ankle injury. Knapik et al. recorded 33,461 instances of parachute jumping with ankle braces and 69,323 instances of parachute jumping without ankle braces, and the results show the injury rate of the latter was 1.83-2.0 times higher than that of the former (6). Wu et al. found that the greater the average angular velocity of inversion and dorsiflexion, the more likely it would cause ankle joint instability on the coronal and sagittal planes. They also found that elastic and semi-rigid ankle braces can significantly reduce rates of injury, but semi-rigid ankle braces are more significantly protective of excessive inversion (4). Willeford et al. found that ankle bandages and ankle braces could significantly reduce the angular displacement of inversion, eversion, plantarflexion, and dorsiflexion of the ankle joint (11).

Despite the protection discussed above, parachute ankle brace designs lack the support of strict mechanical experimental data and a professional theoretical basis. Up to this point, no uniform prophylactic brace against ankle injury exists for when HSPL applications. In this study, a self-designed and self-manufactured novel prophylactic ankle brace [Chinese parachute ankle brace (CPAB)] was developed, and it was compared with elastic ankle braces and semi-rigid ankle braces regarding biomechanical assessments of the ankle joint during HSPL, such as kinetics, kinematics, energy parameters, and the comparison of subjective scores. We present the following article in accordance with the MDAR reporting checklist (available at http://dx.doi.org/10.21037/atm-20-4937).

\section{Methods}

\section{Subjects}

Twenty elite male paratroopers (mean age, $22.56 \pm 3.76 \mathrm{yr}$; mean height, $174.32 \pm 4.58 \mathrm{~cm}$; mean weight, $62.42 \pm 6.93 \mathrm{~kg}$ ) with formal parachute landing training and over $2 \mathrm{yr}$ of parachute jumping experience volunteered for this study. All eligible subjects were healthy and had no history of lower extremity trauma or spinal fractures. None of the subjects had a history of previous surgery of the lower extremities, neurological or joint degenerative diseases, or vestibular or visual disturbances. Each subject was informed of the aims and protocols of this experiment and submitted informed consent before participation. The study was conducted in accordance with the Declaration of Helsinki (as revised in 2013). The ethics committee of the Peking Union Medical College Hospital (XHDW-2015-0006) and informed consent was taken from all the subjects.

\section{Equipment}

The Advanced Mechanical Technology Inc. (AMTI) force 
plate $(1,600 \mathrm{~Hz}$, Watertown, MA, USA) was utilized to measure the vertical ground reaction force (vGRF). The force plate and surrounding floor had similar surface properties to avoid any potential imbalances. A 3D motion capture system $(200 \mathrm{~Hz}$, Vicon, Oxford, UK) was utilized to obtain kinematic data. Reflective surface marker sets were tightly attached to the corresponding bony landmarks. Eight cameras (CMOS, Vicon, Oxford, UK) containing sensors recorded the entire simulated parachute jump in a half-squat posture.

Two commercially available ankle braces and CPAB were used in this experiment: an elastic ankle brace (AQ5261EA, Tokyo, Japan) and a semi-rigid ankle brace (LP787, Seattle, WA, USA). The elastic ankle brace body was composed of an ultra-thin material, the inner shell of which was constructed of high-elastic anti-skid mesh fabric, and the outer shell was constructed of high-elastic shock-absorbing foam. Two straps crossing from the planta in a figure-eight pattern were pressurized and fixed at the lateral and medial malleoli to strengthen ankle joint stability (Figure 1A). The semi-rigid ankle brace contained a U-shaped semirigid metal spring functioning as a "hoop" at the lateral and medial malleoli (Figure 1B).

\section{Fabrication of CPAB}

The CPAB body was composed of a sparsely porous honeycomb-like material called pique fabric (Uniform Hse, Hong Kong, China). An elastic metal spring strip (tensile strength: $800 \mathrm{MPa}$; torsional strength: 28 times $/ 360^{\circ}$; elongation: $3 \%$ ) was constructed on the inner side and adapted to the anatomical outline of the medial malleolus. Also, a special aluminum strip (tensile strength: $\geq 370 \mathrm{MPa}$; yield strength: $\geq 215 \mathrm{MPa}$; elongation: $\geq 12 \%$ ) was constructed on the outer side and adapted to the anatomical outline of the lateral malleolus. The aluminum strip was coated with cotton foam (100\% ethylene-vinyl acetate copolymer) to reduce friction between the ankle joint and CPAB. The strips were the most novel part of the $\mathrm{CPAB}$ design, and their length was $15 \mathrm{~cm}$, their width was $1.2 \mathrm{~cm}$, and their thickness was $0.2 \mathrm{~cm}$. Another special part was the heel pad, which was made of the auxetic material with a negative Poisson ratio (polymer porous polytetrafluoroethylene). When the feet and ankle are impacted by huge ground reaction forces (GRFs), the heel pad can resist excessive deformation and increase comfort. Moreover, impact load conduction is increased and instantaneous impact force is weakened. CPAB is pressurized by an adhesive band at the top and two crossed bands at the back of the foot, which users can utilize adjust the tightness and thereby strengthen the ankle joint stability (Figure 1C,D,E).

\section{Procedure}

Before jumping, each subject jogged for 5 min at a comfortable speed as a warm-up, then performed the HSPL. Upon hearing the order to jump, the subject jumped forward and flexed their lower limbs with their knees, ankles, and forefeet hugging each other and with the plantar parallel to the ground. This was called "three huggings and one parallel" in the teaching material of the China Airborne School. Then they landed on the force plate until their trunk stopped moving and resumed a neutral stance (4). Subjects were evaluated under four different ankle brace conditions (no brace, elastic ankle brace, semi-rigid ankle brace, and $\mathrm{CPAB}$ ) and instructed to start and terminate the drop landing movement in a standing position, to jump off and touch down with both feet, to lean forward with the body while jumping, and finally to stop the fall smoothly in a half-squat position (Figure 2A). Each subject performed this maneuver from three different heights (low: $40 \mathrm{~cm}$, medium: $80 \mathrm{~cm}$, and high: $120 \mathrm{~cm}$ ), undergoing five trials under each condition. The experimental condition order was random to prevent any order effects. Any fatigue effects were mitigated by resting for at least a $60 \mathrm{~s}$ interval between landings under each condition.

Each subject landed on the force plate, which collected GRF signals. A 3D motion capture system was utilized to measure the $3 \mathrm{D}$ position of reflective markers in a global reference frame. Reflective markers were utilized to determine the positions of the bony landmarks as virtual dots (Figure 2B). All bony landmarks were defined as a visual 3D model and analyzed with the Vicon Nexus 2.6 software (C-Motion Inc., Germantown, MD, USA), which was utilized to compute 3D kinematic variables, and the AnyBody model was built to conduct reverse dynamics analysis (Figure 2C). All subjects were briefly asked the same questions after participation, including questions regarding ease of use, quality, comfort, stability, hindrance, and satisfaction. The multiple five-point Likert scale was evaluated by the subjects with five being the best and one being the worst.

\section{Data collection and statistical analysis}

(I) The kinetic parameters: GRF data were measured in 

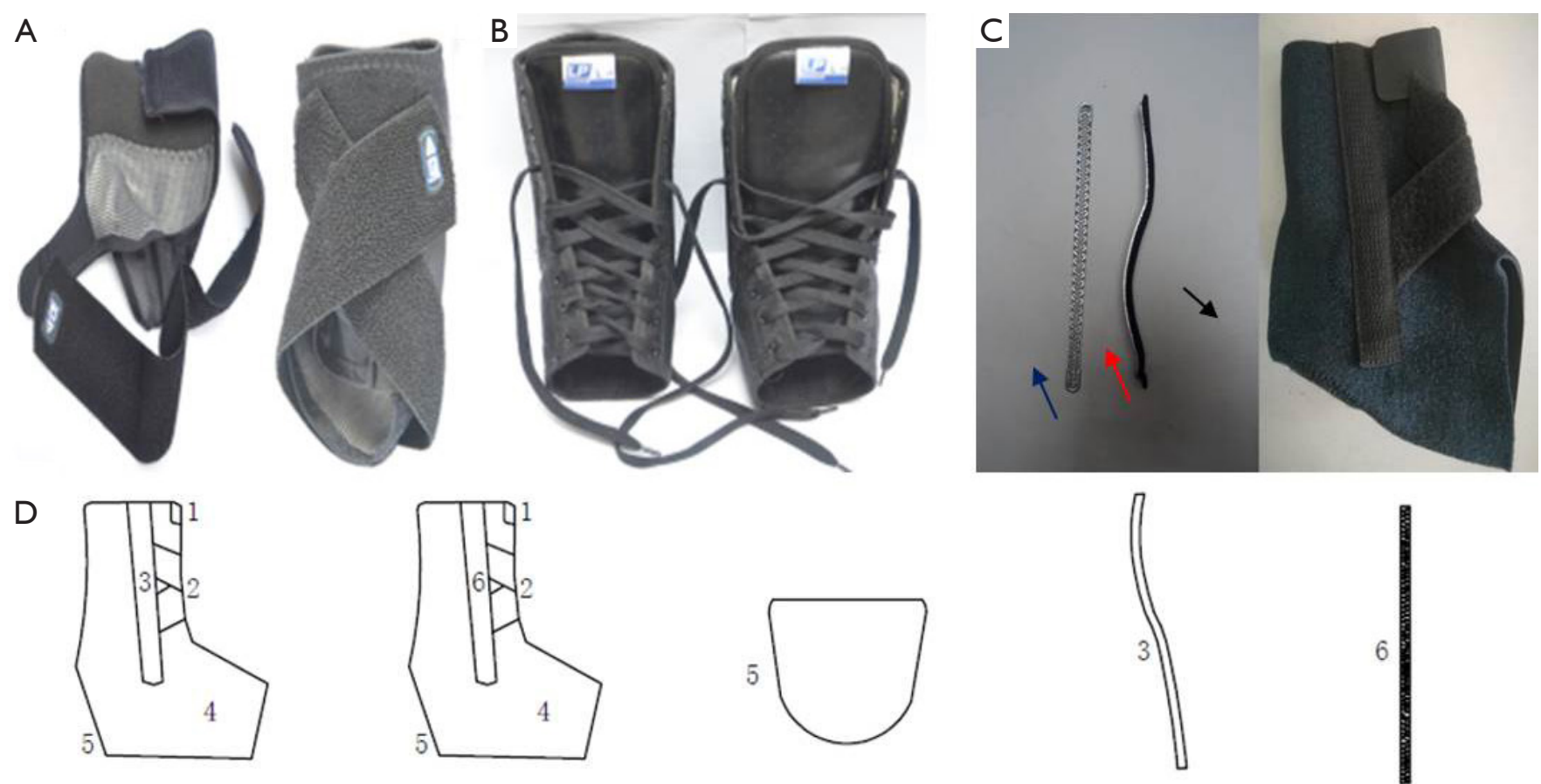

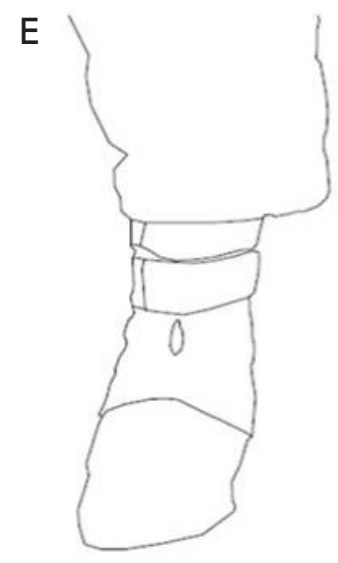

Front view

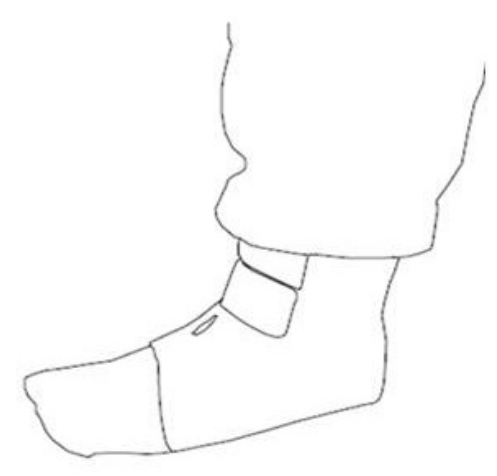

Lateral view
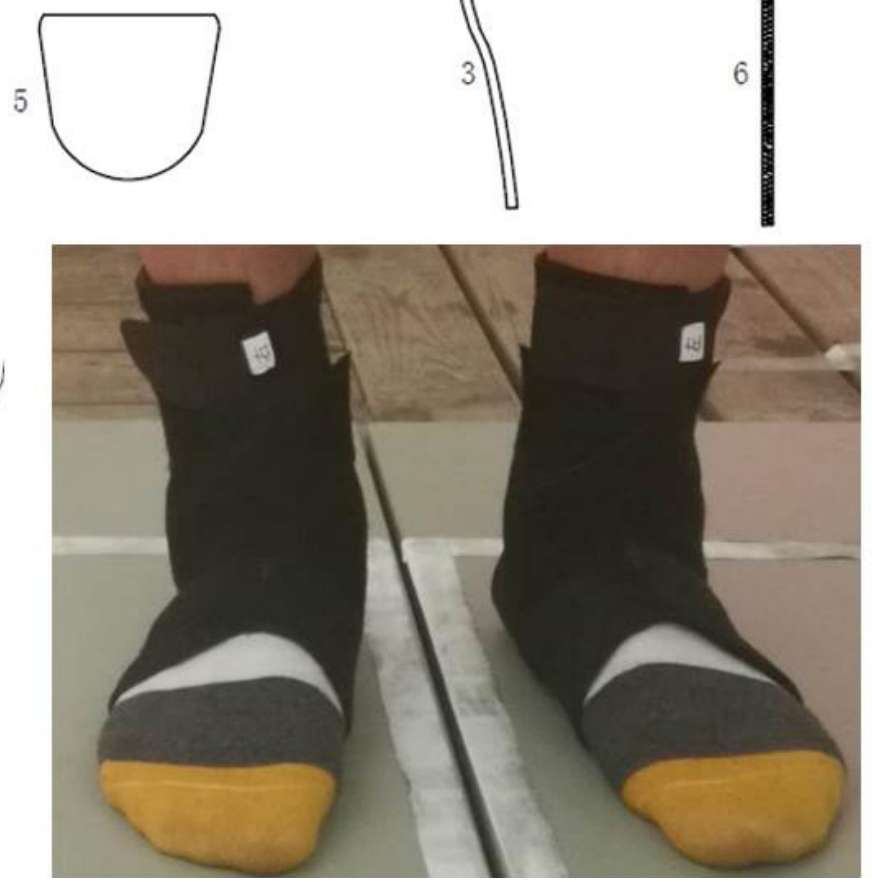

Figure 1 Three types of ankle braces used in the experiment. (A) The elastic ankle brace (AQ5261EA, Tokyo, Japan). (B) The semirigid ankle brace (LP787, Seattle, WA, USA). (C) CPAB. Elastic metal spring strip (blue arrow); an aluminum strip embedded with foam fabric that fits the lateral malleolus profile (red arrow); Heel pad (black arrow). (D) Schematic structure diagram: 1, 2, adhesive bands; 3, aluminum strip; 4, the body of CPAB; 5, heel pad; 6, elastic metal spring strip. (E) Overview of CPAB. CPAB, Chinese parachute ankle brace.

the dominant foot. All vGRF values were normalized to body weight (BW) and the time to peak vGRF (T-PvGRF) started from initial contact with the force plate. The reverse dynamic variables included the maximal plantarflexion moment (MPM) and maximal eversion moment (MEM). (II) The kinematics parameters: angular displacement of maximal dorsiflexion (MDAD), the angular displacement of maximal inversion (MIAD), the angular velocity of maximal dorsiflexion (MDAV), and angular velocity of maximal inversion (MIAV), were calculated with the software (3). The energy parameters: the work and maximum power. From the mechanics perspective, the work refers to the amount of joint power conducted in a certain period time (12), and the calculation formula is as follows: 


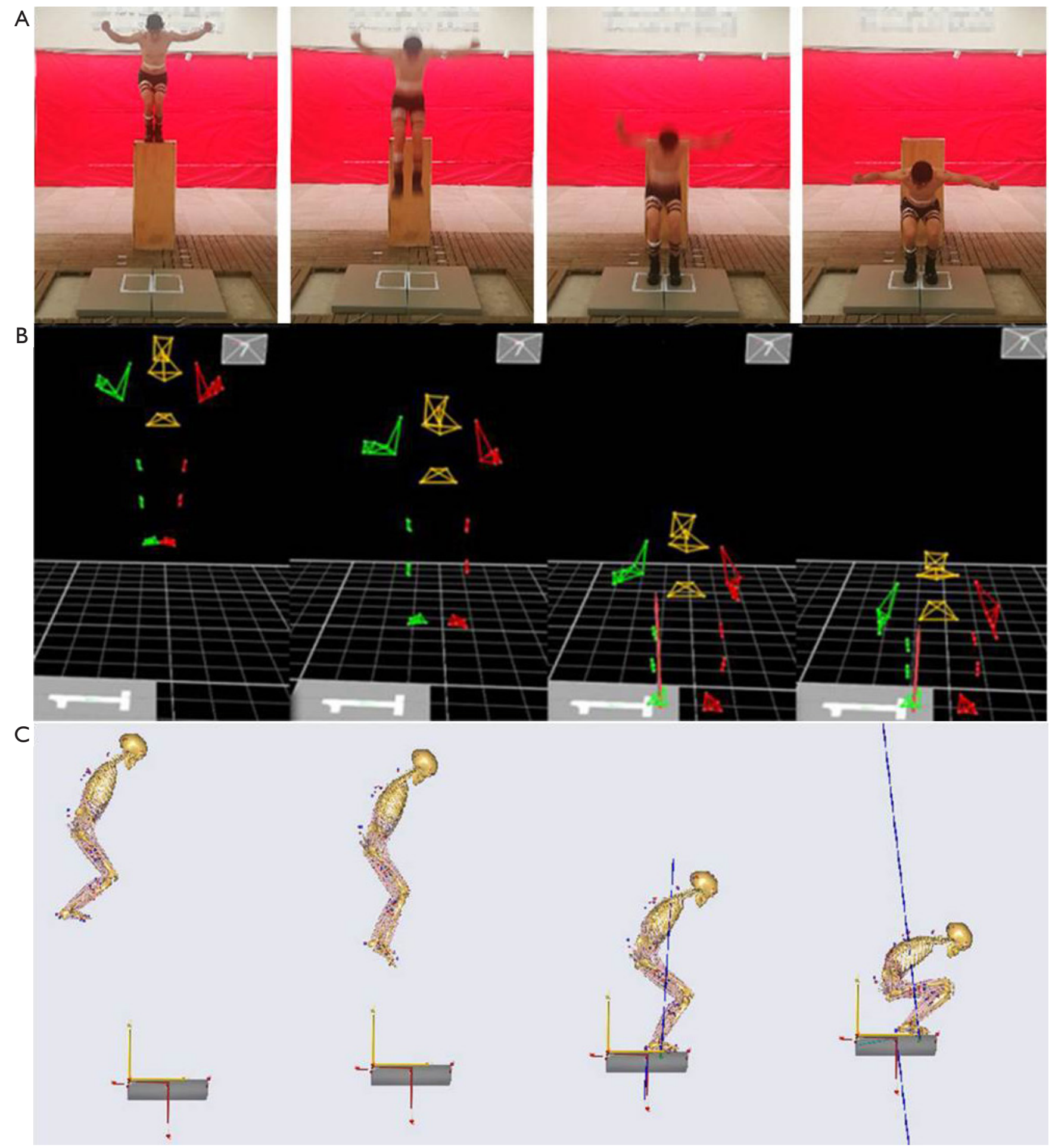

Figure 2 The experimental procedure of HSPL. (A) Each subject performed the HSPL in accordance with a standard protocol. (B) Virtual parachute procedure. Vicon Nexus 2.6 software was used to upload raw data, and confirm a fluent and integrated parachute landing procedure by tracking movement at every time point. (C) The AnyBody musculoskeletal model after muscle loading during HSPL. HSPL, half-squat parachute landing. 
Table 1 Kinetics parameters of ankle joint affected by three different dropping heights and ankle braces during HSPL ( $\mathrm{n}=20$ )

\begin{tabular}{lccccc}
\hline Variables & Heights, cm & No brace & Elastic brace & Semi-rigid brace & CPAB \\
\hline Peak vGRF, BW & 40 & $6.28 \pm 1.51$ & $6.84 \pm 1.29$ & $6.34 \pm 1.32$ & $6.98 \pm 1.20$ \\
& 80 & $7.25 \pm 1.92$ & $7.91 \pm 1.71$ & $7.44 \pm 2.13$ & $7.91 \pm 1.35$ \\
& 120 & $9.28 \pm 2.11$ & $9.98 \pm 2.15$ & $9.64 \pm 2.00$ & $9.87 \pm 2.28$ \\
T-peak vGRF, & 40 & $54.47 \pm 6.03$ & $45.16 \pm 4.76$ & $50.55 \pm 6.16$ & $47.55 \pm 5.59$ \\
ms & 80 & $40.97 \pm 4.74$ & $36.42 \pm 4.17$ & $39.86 \pm 5.26$ & $38.16 \pm 4.20$ \\
& 120 & $28.14 \pm 1.78$ & $22.75 \pm 2.28$ & $28.62 \pm 4.32$ & $22.71 \pm 2.44$ \\
MPM, Nm/kg & 40 & $-3.10 \pm 0.86$ & $-3.08 \pm 0.66$ & $-2.20 \pm 0.89$ & $-2.48 \pm 0.30$ \\
& 80 & $-4.34 \pm 1.32$ & $-4.33 \pm 0.83$ & $-3.31 \pm 0.45$ & $-3.74 \pm 0.43$ \\
& 120 & $-5.17 \pm 1.13$ & $-4.43 \pm 1.25$ & $-3.57 \pm 0.79$ & $-4.02 \pm 1.60$ \\
MEM, Nm/kg $/ \mathrm{kg}, \mathrm{d}, \mathrm{f}$ & $-1.67 \pm 0.31$ & $-1.50 \pm 0.44$ & $-0.88 \pm 0.32$ & $-0.66 \pm 0.21$ \\
& 40 & $-1.97 \pm 0.47$ & $-1.69 \pm 0.36$ & $-0.94 \pm 0.45$ & $-0.81 \pm 0.22$ \\
& 80 & $-2.15 \pm 0.50$ & $-2.14 \pm 0.40$ & $-1.73 \pm 0.28$ & $-1.34 \pm 0.09$ \\
\hline
\end{tabular}

$\S$, significant differences among three dropping heights $(\mathrm{P}<0.05)$; ${ }^{a}$, significant differences between the no-brace group and elastic brace group $(\mathrm{P}<0.05)$; ${ }^{\mathrm{b}}$, significant differences between the no-brace group and semi-rigid brace group $(\mathrm{P}<0.05)$; ${ }^{\mathrm{c}}$, significant differences between the no-brace group and CPAB group $(P<0.05)$; ${ }^{d}$, significant differences between the elastic brace group and semi-rigid brace group $(\mathrm{P}<0.05)$; ${ }^{e}$, significant differences between the elastic brace group and CPAB group $(\mathrm{P}<0.05)$; ${ }^{f}$, significant differences between the semi-rigid brace group and CPAB group $(\mathrm{P}<0.05)$. HSPL, half-squat parachute landing; $\mathrm{CPAB}$, Chinese parachute ankle brace; vGRF, vertical ground reaction force; T-peak vGRF, time to peak vGRF; MPM, maximum plantar moment; MEM, maximum eversion moment.

$$
\text { Work }=\int_{t 2}^{t 1} P(t) \cdot d t
$$

$P$ represents joint power, and $t_{1}$ and $t_{2}$ represent the start and end time points. The work done by the ankle joint from initial contact with the force plate to buffering completion is negative work, namely energy absorption. Joint power $=$ angular velocity $\times$ joint moment, which can be obtained by Anybody reverse dynamics analysis. In this study, dorsiflexion and inversion were stipulated to be positive, while plantarflexion and eversion were negative.

Data are representative of these experiments and are shown as the means \pm standard deviation (SD). Two treatment groups were compared via the $t$-test of Students. Multiple group comparisons were performed via a two-way analysis of variance with Tukey's post hoc test. Statistical analysis was conducted using GraphPad Prism 7.0 software, and statistical significance was declared as $\mathrm{P}<0.05$.

\section{Results}

All participants completed the experiment and none were injured during testing. According to previous research, ankle joints were prone to instability on the coronal and sagittal planes, namely, excessive inversion or dorsiflexion.
During HSPL, the subject always maintained standard posture (the feet and medial malleoli hugged together), and feet were parallel to the ground when landing, so excessive eversion and plantarflexion would not occur. Therefore, this experiment mainly focused on inversion and dorsiflexion.

\section{The assessment of kinetic parameters}

Table 1 shows that increasing heights significantly increased peak vGRF, MPM, and MEM $(\mathrm{P}<0.01)$, while T-PvGRF significantly decreased $(\mathrm{P}<0.01)$ and $\mathrm{T}-\mathrm{PvGRF}$ gradually became smaller. The T-PvGRF was often very short, whereas the time to complete the landing process was much longer. In other words, the ankle joint was not dorsiflexed enough and did not have a complete buffer at peak vGRF, and the latter and the muscles could not properly absorb the force. Whole-body instability during landing may result in a shorter T-PvGRF because, when the body was unstable, the center of gravity will change from one position to another.

When compared with the no-brace condition, the use of three ankle braces can all increase peak vGRF, but no significant difference existed $(\mathrm{P}=0.237)$. Wearing an elastic ankle brace and CPAB both increase peak vGRF by about $10 \%$, while a semi-rigid ankle brace had no significant effect 
on peak vGRF $(\mathrm{t}=0.106, \mathrm{P}=0.564)$. Figure 3 shows that the use of three ankle braces can all reduce T-PvGRF $(\mathrm{P}<0.01)$ and an elastic ankle brace and CPAB reduced T-PvGRF with 6-10 $\mathrm{ms}$ at the dropping height of $120 \mathrm{~cm}$. Wearing ankle braces can also reduce MPM and MEM, according to the degree of MPM reduction, semi-rigid brace $(t=5.309$, $\mathrm{P}<0.01), \mathrm{CPAB}(\mathrm{t}=3.816, \mathrm{P}<0.01)$ and elastic brace $(\mathrm{t}=2.455$, $\mathrm{P}<0.05)$ were followed. Both $\mathrm{CPAB}$ and a semi-rigid ankle brace can significantly reduce MEM, and CPAB showed a more significant effect $(\mathrm{t}=3.449, \mathrm{P}<0.01)$.

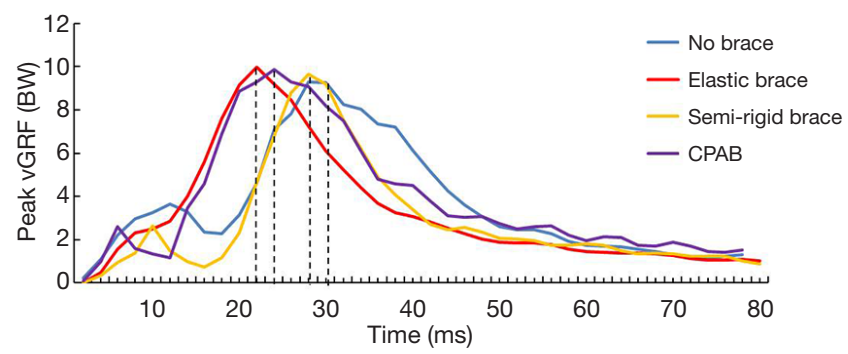

Figure 3 Time-dependent curves of vGRF during HSPL under different ankle braces conditions at $120 \mathrm{~cm}$. vGRF, vertical ground reaction force; HSPL, half-squat parachute landing.

\section{The assessment of kinematics parameters}

Table 2 shows that the dropping heights had a significant effect on MDAD $(\mathrm{P}<0.01)$, MIAD $(\mathrm{P}<0.01)$, MDAV $(\mathrm{P}<0.01)$, and MIAV $(\mathrm{P}<0.01)$. When compared with the nobrace condition, wearing ankle braces can decrease MDAV and MIAV with no significant differences. According to the degree of MIAD reduction from large to small, $\mathrm{CPAB}$ was $(\mathrm{t}=17.97, \mathrm{P}<0.01)$, semi-rigid brace was $(\mathrm{t}=15.12, \mathrm{P}<0.01)$, and an elastic brace was $(\mathrm{t}=4.201, \mathrm{P}<0.01)$. When compared with the elastic brace, $\mathrm{CPAB}$, and the semi-rigid brace were more able to limit inversion, as they can each reduce MDAD by about $8^{\circ}(\mathrm{t}=3.974, \mathrm{P}<0.01)$ and $15^{\circ}(\mathrm{t}=8.264$, $\mathrm{P}<0.01)$.

Collectively, three ankle braces can restrict dorsiflexion and inversion during landings and maintain ankle joint stability on the coronal and sagittal planes, while $\mathrm{CPAB}$ provided greater inversion limitations than the other two ankle braces.

\section{The assessment of energy parameters}

Table 3 shows that increasing dropping heights significantly increased the work and maximum power $(\mathrm{P}<0.01)$. When compared with the no-brace condition and wearing an

Table 2 Kinematics parameters of ankle joint affected by three different dropping heights and ankle braces during HSPL ( $\mathrm{n}=20$ )

\begin{tabular}{lccccc}
\hline Variables & Heights, cm & No brace & Elastic brace & Semi-rigid brace & CPAB \\
\hline MDAD, ${ }^{\circ}$ & 40 & $18.97 \pm 3.76$ & $18.12 \pm 3.28$ & $15.22 \pm 4.01$ & $16.94 \pm 2.15$ \\
& 80 & $26.08 \pm 4.03$ & $25.01 \pm 4.10$ & $22.83 \pm 5.16$ & $25.64 \pm 3.81$ \\
& 120 & $43.31 \pm 7.67$ & $42.30 \pm 7.24$ & $29.48 \pm 6.38$ & $36.66 \pm 8.02$ \\
MIAD, ${ }^{\circ}$ & 40 & $8.17 \pm 0.25$ & $8.00 \pm 0.49$ & $6.89 \pm 0.87$ & $6.83 \pm 0.41$ \\
& 80 & $13.19 \pm 0.93$ & $13.02 \pm 1.19$ & $9.50 \pm 0.27$ & $9.32 \pm 0.43$ \\
& 120 & $27.03 \pm 1.59$ & $29.70 \pm 3.02$ & $17.42 \pm 3.24$ & $15.61 \pm 4.75$ \\
MDAV, ${ }^{\circ} / \mathrm{s}$ & 40 & $554.38 \pm 118.57$ & $512.83 \pm 139.50$ & $-2.20 \pm 0.89$ & $469.24 \pm 70.39$ \\
& 80 & $763.50 \pm 250.40$ & $637.85 \pm 194.35$ & $-3.31 \pm 0.45$ & $620.37 \pm 125.20$ \\
& 120 & $1,021.93 \pm 330.29$ & $929.17 \pm 312.13$ & $-3.57 \pm 0.79$ & $859.77 \pm 330.93$ \\
MIAV, ${ }^{\circ} / \mathrm{s}$ & 40 & $339.83 \pm 21.39$ & $297.92 \pm 18.17$ & $249.99 \pm 22.57$ & $286.03 \pm 20.31$ \\
& 80 & $472.33 \pm 114.27$ & $443.41 \pm 145.09$ & $395.45 \pm 68.34$ & $431.49 \pm 116.39$ \\
& 120 & $588.42 \pm 195.45$ & $564.72 \pm 216.36$ & $500.16 \pm 264.36$ & $533.97 \pm 129.04$ \\
\hline
\end{tabular}

$\S$, significant differences among three dropping heights $(P<0.05) ;{ }^{b}$, significant differences between the no-brace group and semi-rigid brace group $(P<0.05) ;{ }^{c}$, significant differences between the no-brace group and CPAB group $(P<0.05)$; ${ }^{d}$, significant differences between the elastic brace group and semi-rigid brace group $(P<0.05)$; ${ }^{e}$, significant differences between the elastic brace group and $C P A B$ group $(\mathrm{P}<0.05)$. HSPL, half-squat parachute landing; $\mathrm{CPAB}$, Chinese parachute ankle brace; MDAD, angular displacement of maximal dorsiflexion; MIAD, angular displacement of maximal inversion; MDAV, angular velocity of maximal dorsiflexion; MIAV, angular velocity of maximal inversion. 
Table 3 Energy parameters of ankle joint affected by three different dropping heights and ankle braces during HSPL ( $\mathrm{n}=20)$

\begin{tabular}{lccccc}
\hline Variables & Heights, cm & No brace & Elastic brace & Semi-rigid brace & CPAB \\
\hline Peak power, W & 40 & $-8.49 \pm 1.33$ & $-8.14 \pm 0.76$ & $-6.42 \pm 0.25$ & $-5.80 \pm 0.23$ \\
& 80 & $-21.34 \pm 8.06$ & $-19.25 \pm 6.88$ & $-17.57 \pm 8.14$ & $-17.60 \pm 8.50$ \\
& 120 & $-44.03 \pm 16.72$ & $-41.82 \pm 13.21$ & $-26.17 \pm 9.52$ & $-24.77 \pm 7.21$ \\
Work, J/kg & 40 & $-0.75 \pm 0.13$ & $-0.78 \pm 0.10$ & $-0.45 \pm 0.09$ & $-0.29 \pm 0.06$ \\
& 80 & $-1.28 \pm 0.34$ & $-1.27 \pm 0.35$ & $-1.10 \pm 0.30$ & $-1.08 \pm 0.34$ \\
& 120 & $-1.70 \pm 0.37$ & $-1.68 \pm 0.42$ & $-1.59 \pm 0.36$ & $-1.43 \pm 0.22$ \\
\hline
\end{tabular}

$\S$, significant differences among three dropping heights $(\mathrm{P}<0.05) ;{ }^{b}$, significant differences between the no-brace group and semi-rigid brace group $(\mathrm{P}<0.05) ;{ }^{c}$, significant differences between the no-brace group and CPAB group $(\mathrm{P}<0.05)$; ${ }^{d}$, significant differences between the elastic brace group and semi-rigid brace group $(P<0.05)$; ${ }^{e}$, significant differences between the elastic brace group and $C P A B$ group $(\mathrm{P}<0.05)$. HSPL, half-squat parachute landing; CPAB, Chinese parachute ankle brace.

Table 4 The score results of three kinds of ankle braces $(n=20)$

\begin{tabular}{|c|c|c|c|c|c|}
\hline Variables & Elastic brace & Semi-rigid brace & CPAB & $\mathrm{F}$ & $P$ value \\
\hline Ease of use & $3.70 \pm 0.38$ & $3.90 \pm 0.12$ & $4.10 \pm 0.27$ & 10.36 & $\mathrm{P}<0.01^{\mathrm{b}}$ \\
\hline Quality & $3.60 \pm 0.40$ & $4.10 \pm 0.28$ & $3.80 \pm 0.12$ & 15.03 & $\mathrm{P}<0.01^{\mathrm{a}, \mathrm{c}}$ \\
\hline Comfort & $4.00 \pm 0.15$ & $3.00 \pm 0.33$ & $3.70 \pm 0.26$ & 79.40 & $\mathrm{P}<0.01^{\mathrm{a}, \mathrm{b}, \mathrm{c}}$ \\
\hline Stability & $3.10 \pm 0.42$ & $4.00 \pm 0.22$ & $3.60 \pm 0.23$ & 43.93 & $\mathrm{P}<0.01^{\mathrm{a}, \mathrm{b}, \mathrm{c}}$ \\
\hline Satisfaction & $3.30 \pm 0.43$ & $2.70 \pm 0.59$ & $4.40 \pm 0.26$ & 74.26 & $\mathrm{P}<0.01^{\mathrm{a}, \mathrm{b}, \mathrm{c}}$ \\
\hline Total scores & $21.91 \pm 3.25$ & $19.20 \pm 2.99$ & $23.37 \pm 3.08$ & 9.267 & $\mathrm{P}<0.01^{\mathrm{a}, \mathrm{c}}$ \\
\hline
\end{tabular}

${ }^{a}$, significant differences between the elastic brace group and semi-rigid brace group $(P<0.05) ;{ }^{b}$, significant differences between the elastic brace group and CPAB group $(\mathrm{P}<0.05)$; ${ }^{c}$, significant differences between the semi-rigid brace group and $\mathrm{CPAB}$ group $(\mathrm{P}<0.05)$. $\mathrm{CPAB}$, Chinese parachute ankle brace.

elastic brace, no significant differences existed in ankle work and maximum power $(\mathrm{P}>0.05)$, whereas the use of the semirigid brace or $\mathrm{CPAB}$ decreased the work $(\mathrm{t}=3.331, \mathrm{P}<0.01$; $\mathrm{t}=5.107, \mathrm{P}<0.01)$ and maximum power $(\mathrm{t}=6.711, \mathrm{P}<0.01$; $\mathrm{t}=7.237, \mathrm{P}<0.01)$. Therefore, subjects wearing $\mathrm{CPAB}$ can effectively reduce the amount of work and power, further allowing the ankle to absorb less energy.

\section{The assessment of the subjective score}

Table 4 shows the subjective scores of the subjects of the three ankle braces were all statistically significant $(\mathrm{P}<0.01)$, and the total scores from low to high were semi-rigid

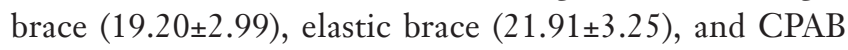
(23.37 \pm 3.08$)$. Eighty-five percent of the subjects agreed that $\mathrm{CPAB}$ restricted inversion and eversion more effectively than the elastic brace, and was more comfortable and soft than the semi-rigid ankle brace. Therefore, $\mathrm{CPAB}$ combined the advantages of the other two ankle braces, with good comfort, ease of use, high stability, and light constriction. All the subjects were satisfied with the appearance, function, and comfort of CPAB.

\section{Discussion}

Ankle injuries mainly occurred during parachute training and landing, and even led to irreversible injuries, which seriously affected army combat capability (13-15). Therefore, paratrooper ankle protection is an urgent problem that needs to be solved. Luippold et al. developed an out-the-boot parachute ankle brace, which reduced ankle injury rates by $50 \%$ (16). The U.S. Department of Defence Center for Health Promotion and Preventative Medicine suggested paratroopers should wear semi-rigid 
ankle braces in 2010 (17). In Britain, Australia, Germany, and other developed countries, internal ankle support was widely used by paratroopers as necessary protective equipment $(4,10,18)$. Although air-filled ankle braces, EVC foam braces, and high-elastic-fiber ankle socks had been developed successively in China, no relevant report exists on whether or not they can effectively prevent ankle injuries $(4,5,7,19)$. According to the epidemiological survey and preliminary biomechanical research conducted for this study, the injury mechanism was found to be excessive dorsiflexion and inversion of the ankle joint during HSPL, and it was also found that wearing ankle braces can effectively restrict ankle joint motion stability on the sagittal and coronal planes $(4,7)$. Therefore, in this study, a built-in ankle brace suitable for Chinese paratroopers was designed and fabricated. Moreover, a large peak vGRF was the fundamental ankle injury cause in parachute landings (20). The ideal novel parachute ankle brace can not only avoid excessive dorsiflexion and inversion during parachute landings but also effectively reduce the instantaneous GRF impact.

According to the anatomy of ankle joints, the strong deltoid ligament is on the medial, while the lateral calcaneofibular ligament, anterior talofibular ligament, and posterior talofibular ligament on the lateral are relatively weak (21). Great GRF impacts easily caused the lateral collateral ligament injury and even ruptured during parachute landings. The novel military parachute ankle brace $\mathrm{CPAB}$ design should be complementary to the asymmetric anatomic ankle joint structure. Since the lateral ligaments are weak, greater strength materials should be used on the outside of $\mathrm{CPAB}$ to protect the ankle and vice versa. Collectively, in the $\mathrm{CPAB}$, the rigidity and strength of the lateral malleolus side should be greater than that of the medial malleolus side.

$\mathrm{CPAB}$ design should also consider appearance, materials, and biomechanics. Several innovative points in $\mathrm{CPAB}$ are as follows: (I) high security. Hinged structure reinforcement was widely used in the medial and lateral malleoli of ankle braces, and the curved protection body conforming to the anatomical structure of the medial and lateral malleoli was rare $(22,23)$. The hard aluminum strip was consistent with the anatomical contour of the lateral malleolus and effectively limited inversion. Moreover, the inside of the aluminum strip was covered with foam fabric to reduce friction between the lateral malleolus and $\mathrm{CPAB}$, further protecting the skin there and avoiding severe stress from the aluminum support. (II) Heel protection. Preliminary biomechanical research showed that peak vGRF can reach more than 10 times that of BW (4). The heel pad was utilized to prolong buffer time, reduce peak vGRF, scatter sole pressure, and change sole pressure distribution. (III) Great comfort level. CPAB raw material (pique fabric) reduced relative slip between the protection device and human skin and improved protection reliability. According to studies on textile moisture absorption (24), pique fabric was more air permeable, drier, and washable than knitted fabric. van den Bekerom et al. used silicone membrane as the main ankle brace material, which was lightweight and breathable, whereas the surface was relatively smooth, with little friction and prone to slippage and displacement during strenuous exercises (25).

In this study, a gradable experimental jumping platform with heights of 40,80 , and $120 \mathrm{~cm}$ was utilized to obtain increasing landing speeds, since the actual height for Chinese parachuting ground training is less than $150 \mathrm{~cm}$. In addition to kinetic and kinematic parameters, the advanced Anybody musculoskeletal model and reverse dynamics analysis could calculate $3 \mathrm{D}$ net torque, power, and work of the ankle joint. Many studies showed that vGRF increased significantly as the jumping height increased (12,26-29), indicating that GRF suffered by subjects was positively correlated with the height. While T-PvGRF reflected landing buffering capacity in the early landing stage, this study showed that increasing the height significantly decreased T-PvGRF, which demonstrated body instability and center-of-mass deviation. However, the T-PvGRF increase did not mean that the landing was more stable; the study of simulated sideways roll parachute landing showed that the T-PvGRF value didn't vary with the height (30). Therefore, whether or not a correlation existed between T-PvGRF and jumping height remained to be verified by future experiments. Increasing height had a significant influence on the sagittal MPM, MDAD, MDAV, and coronal MEM, MIAD, and MIAV of the ankle joint, as well as the work and power. The ankle passively generated MPM and MEM to resist external torques (dorsiflexion moment and inversion moment) generated by GRF. Peak torque can be used as a sensitive indicator to evaluate muscle strength (31). The ankle absorbed the energy and the plantar muscles applied the work to reduce the GRF impact and increase the buffer. Since greater angular velocity meant greater joint momentum, the ankle joint may absorb more energy and transfer energy along the lower limbs to the knee, hip, and spine (32).

In this experiment, the effect of wearing $\mathrm{CPAB}$ on the 
kinetic, kinematic, and energy parameters of the ankle joint was more significant than that of wearing an elastic ankle brace or a semi-rigid ankle brace. Thanks to the special lateral support structure, $\mathrm{CPAB}$ provided greater inversion limitation than the other two ankle braces. When compared with the wrapping elastic brace, the laceup straps of the semi-rigid brace and the adhesive bands of CPAB more effectively limited dorsiflexion. Niu et al. investigated the effects of different ankle braces on lower limb biomechanics during HSPL and revealed that rigid ankle brace usage could enhance muscle activity (19). Some scholars acknowledged that elastic deformation of the support structure on both sides of the ankle brace provided an eversion moment to overcome inversion and reduced the work (33). Based on Janssen et al.'s (9) Constructs of Subjective Factors of Brace Use, the questionnaires were designed using the Likert 5-point scale for each evaluation index, including ease of use, quality, comfort, stability, hindrance, and satisfaction. In general, the CPAB score was considered superior to the other two braces. The comfort $(3.00 \pm 0.33)$ and satisfaction $(2.70 \pm 0.59)$ of the semi-rigid ankle brace were low due to the sharp edge and hard material. However, the low hindrance $(2.90 \pm 0.18)$ and stability $(3.10 \pm 0.42)$ scores of the elastic brace were attributed to the lack of rigid support structure and special protection for the medial and lateral malleoli. Eighty-five percent of the subjects agreed that $\mathrm{CPAB}$ was more effective for ankle inversion and eversion restriction than the elastic brace, and more comfortable and soft than the semi-rigid brace. In the future, more paratroopers will be equipped with $\mathrm{CPAB}$, and in turn, more subjective feedback will be acquired.

Our research also had some shortcomings: (I) the biomechanical experiment was based on the conclusion of our previous studies, although wearing elastic and semirigid ankle braces both had no significant effect on the extorsion and intorsion of ankle joint, whether CPAB also had no significant limitation on the lateral rotation motion of ankle joint remained to be proved. (II) According to the known holistic synergy theory of the lower extremity, whether the restriction of the ankle joint increased the energy consumption or injury of the knee and hip joint remained to be further explored.

$\mathrm{CPAB}$, as an ankle brace for parachuting, was still preliminary, and it would sustain external forces, including huge GRF at the moment of landing and continuous small stress or strain at all directions. Therefore, the CPAB should have good mechanical properties which need to be tested in the future research, including the shaping test, tensile test, anti-fatigue test, fabric permeability test and fabric friction test, etc.

\section{Conclusions}

A novel military parachute ankle brace, $\mathrm{CPAB}$, was designed and prepared according to the biomechanical characteristics of excessive dorsiflexion and inversion during paratroopers' HSPL. Biomechanical tests showed that increasing the dropping height resulted in greater peak vGRF and energy parameters, which may lead to ligament damage or even fractures during parachute landings. The CPAB more markedly restricted the motion of the ankle joint on the coronal and sagittal planes than the elastic and semi-rigid ankle braces. Therefore, the CPAB had the advantages of a novel appearance, high efficiency, and superior comfort, providing a reliable choice for parachute jumping and training in China.

\section{Acknowledgments}

This work was supported by the Innovation Project of Peking Union Medical College Graduate Student (20191002-11). The authors would like to thank all subjects who participated in this study.

Funding: None.

\section{Footnote}

Reporting Checklist: The authors have completed the MDAR reporting checklist. Available at http://dx.doi.org/10.21037/ atm-20-4937

Data Sharing Statement: Available at http://dx.doi. org/10.21037/atm-20-4937

Peer Review File: Available at http://dx.doi.org/10.21037/ atm-20-4937

Conflicts of Interest: All authors have completed the ICMJE uniform disclosure form (available at http://dx.doi. org/10.21037/atm-20-4937). The authors have no conflicts of interest to declare.

Ethical Statement: The authors are accountable for all aspects of the work in ensuring that questions related to the accuracy or integrity of any part of the work are 
appropriately investigated and resolved. The study was conducted in accordance with the Declaration of Helsinki (as revised in 2013). The ethics committee of the Peking Union Medical College Hospital (XHDW-2015-0006) and informed consent was taken from all the subjects.

Open Access Statement: This is an Open Access article distributed in accordance with the Creative Commons Attribution-NonCommercial-NoDerivs 4.0 International License (CC BY-NC-ND 4.0), which permits the noncommercial replication and distribution of the article with the strict proviso that no changes or edits are made and the original work is properly cited (including links to both the formal publication through the relevant DOI and the license). See: https://creativecommons.org/licenses/by-nc-nd/4.0/.

\section{References}

1. Yeh RW, Valsdottir LR, Yeh MW, et al. Parachute use to prevent death and major trauma when jumping from aircraft: randomized controlled trial. BMJ 2018;363:k5094.

2. Ekeland A. Injuries in military parachuting: a prospective study of 4499 jumps. Injury 1997;28:219-22.

3. Dhar D. Retrospective study of injuries in military parachuting. Med J Armed Forces India 2007;63:353-5.

4. Wu D, Zheng C, Wu J, et al. Prophylactic ankle braces and the kinematics and kinetics of half-squat parachute landing. Aerosp Med Hum Perform 2018;89:141-6.

5. Wu D, Zheng C, Wu J, et al. Protective knee braces and the biomechanics of the half-squat parachute landing. Aerosp Med Hum Perform 2018;89:26-31.

6. Knapik J, Steelman R. Risk factors for injuries during military static-line airborne operations: a systematic review and meta-analysis. J Athl Train 2016;51:962-80.

7. Li Y, Wu J, Zheng C, et al. The effect of landing surface on the plantar kinetics of chinese paratroopers using halfsquat landing. J Sports Sci Med 2013;12:409-13.

8. Harmon KG, Drezner JA, Gammons M, et al. American Medical Society for Sports Medicine position statement: concussion in sport. Br J Sports Med 2013;47:15-26.

9. Janssen K, Van Den Berg A, Van Mechelen W, et al. User survey of 3 ankle braces in soccer, volleyball, and running: which brace fits best? J Athl Train 2017;52:730-7.

10. Newman TM, Gay MR, Buckley WE. Prophylactic ankle bracing in military settings: a review of the literature. Mil Med 2017;182:e1596-602.

11. Willeford K, Stanek JM, McLoda TA. Collegiate football players' ankle range of motion and dynamic balance in braced and self-adherent-taped conditions. J Athl Train 2018;53:66-71.

12. Yeow CH, Lee PV, Goh JC. Effect of landing height on frontal plane kinematics, kinetics and energy dissipation at lower extremity joints. J Biomech 2009;42:1967-73.

13. Abt JP, Sell TC, Lovalekar MT, et al. Injury epidemiology of U.S. Army Special Operations forces. Mil Med 2014;179:1106-12.

14. Kucera KL, Marshall SW, Wolf SH, et al. Association of injury history and incident injury in cadet basic military training. Med Sci Sports Exerc 2016;48:1053-61.

15. Lovalekar MT, Abt JP, Sell TC, et al. Descriptive epidemiology of musculoskeletal injuries in the Army 101st Airborne (Air Assault) Division. Mil Med 2016;181:900-6.

16. Luippold RS, Sulsky SI, Amoroso PJ. Effectiveness of an external ankle brace in reducing parachuting-related ankle injuries. Inj Prev 2011;17:58-61.

17. Bullock SH, Jones BH, Gilchrist J, et al. Prevention of physical training-related injuries recommendations for the military and other active populations based on expedited systematic reviews. Am J Prev Med 2010;38:S156-81.

18. Knapik JJ, Spiess A, Swedler DI, et al. Systematic review of the parachute ankle brace: injury risk reduction and cost effectiveness. Am J Prev Med 2010;38:S182-8.

19. Niu W, Wang Y, Yao J, et al. Consideration of gender differences in ankle stabilizer selection for halfsquat parachute landing. Aviat Space Environ Med 2011;82:1118-24.

20. Niu W, Feng T, Wang L, et al. Effects of prophylactic ankle supports on vertical ground reaction force during landing: a meta-analysis. J Sports Sci Med 2016;15:1-10.

21. Vega J, Malagelada F, Manzanares Cespedes MC, et al. The lateral fibulotalocalcaneal ligament complex: an ankle stabilizing isometric structure. Knee Surg Sports Traumatol Arthrosc 2020;28:8-17.

22. Alfuth M, Klein D, Koch R, et al. Biomechanical comparison of 3 ankle braces with and without free rotation in the sagittal plane. J Athl Train 2014;49:608-16.

23. Sætersdal C, Fevang JM, Engesæter LB. Inferior results with unilateral compared with bilateral brace in Ponsetitreated clubfeet. J Child Orthop 2017;11:216-22.

24. Arnold BL, Docherty CL. Bracing and rehabilitation-what's new. Clin Sports Med 2004;23:83-95.

25. van den Bekerom MP, van Kimmenade R, Sierevelt IN, et al. Randomized comparison of tape versus semi-rigid and versus lace-up ankle support in the treatment of acute lateral ankle ligament injury. Knee Surg Sports Traumatol Arthrosc 2016;24:978-84. 
26. Quatman CE, Ford KR, Myer GD, et al. Maturation leads to gender differences in landing force and vertical jump performance: a longitudinal study. Am J Sports Med 2006;34:806-13.

27. Barker LA, Harry JR, Mercer JA. Relationships between countermovement jump ground reaction forces and jump height, reactive strength index, and jump time. J Strength Cond Res 2018;32:248-54.

28. Phillips JH, Flanagan SP. Effect of ankle joint contact angle and ground contact time on depth jump performance. J Strength Cond Res 2015;29:3143-8.

29. Harry JR, James CR, Dufek JS. Weighted vest effects on impact forces and joint work during vertical jump landings

Cite this article as: Zhou $\mathrm{X}, \mathrm{Wu} \mathrm{D}, \mathrm{Wu} \mathrm{X}, \mathrm{Li} \mathrm{Z}$, Yan B, Liang L, He Y, Liu Y. A novel prophylactic Chinese parachute ankle brace. Ann Transl Med 2021;9(4):318. doi: 10.21037/atm20-4937 in men and women. Hum Mov Sci 2019;63:156-63.

30. Whitting JW, Steele JR, Jaffrey MA, et al. Parachute landing fall characteristics at three realistic vertical descent velocities. Aviat Space Environ Med 2007;78:1135-42.

31. Kim H, Son SJ, Seeley MK, et al. Kinetic Compensations due to chronic ankle instability during landing and jumping. Med Sci Sports Exerc 2018;50:308-17.

32. Hueber GA, Hall EA, Sage BW, et al. Prophylactic bracing has no effect on lower extremity alignment or functional performance. Int J Sports Med 2017;38:637-43.

33. Fatoye F, Haigh C. The cost-effectiveness of semi-rigid ankle brace to facilitate return to work following first-time acute ankle sprains. J Clin Nurs 2016;25:1435-43. 